\title{
Continuous Stirred Tank Reactor Fault Detection Using Higher Degree Cubature Kalman Filter
}

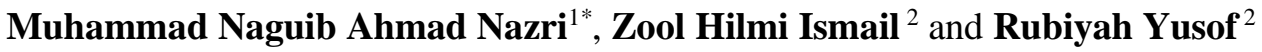 \\ ${ }^{1}$ Malaysia-Italy Design Institute, Universiti Kuala Lumpur, 56100 Kuala Lumpur, Malaysia. \\ ${ }^{2}$ Malaysia-Japan International Institute of Tech, Universiti Teknologi Malaysia, 54100 Kuala Lumpur, Malaysia.
}

"Corresponding author: mnaguib@ unikl.edu.my, Tel: 603-91795000, Fax: 603-91795001

\begin{abstract}
Continuous Stirred Tank Reactor (CSTR) plays a major role in chemical industries, it ensures the process of mixing reactants according to the attended specification to produce a specific output. It is a complex process that usually represent with nonlinear model for benchmarking. Any abnormality, disturbance and unusual condition can easily interrupt the operations, especially fault. And this problem need to detect and rectify as soon as possible. A good knowledge based fault detection using available model require a good error residual between the measurement and the estimated state. Kalman filter is an example of a good estimator, and has been exploited in many researches to detect fault. In this paper, Higher degree Cubature Kalman Filter (HDCKF) is proposed as a method for fault detection by estimation the current state. Cubature Kalman filter (CKF) is an extension of the Kalman filter with the main purpose is to estimate process and measurement state with high nonlinearities. It is based on spherical radial integration to estimate current state by generating cubature points with specific value. Conventional CKF use 3rd degree spherical and 3rd degree radial, here we implement Higher Degree CKF (HDCKF) to have better accuracy as compared to conventional CKF. High accuracy is required to ensure no false alarm is detected and furthermore good computational cost will improve its detection. Finally, a numerical example of CSTR fault detection using HDCKF is presented. Implementation of HDCKF for fault detection is compared with other filter to show effective results.
\end{abstract}

Keywords: continuous stirred tank reactor, cubature kalman filter, estimator, fault detection, nonlinear system.

(C) 2019 Penerbit UTM Press. All rights reserved

Article History: received 4 September 2019; accepted 1 December 2019; published 24 December 2019.

\section{INTRODUCTION}

Continuous Stirred Tank Reactor (CSTR) represent a general description of the actual chemical industry process. It is design with large number of sensors and actuators to monitor and control the process. With a complex build and a nonlinear characteristic these system is susceptible to faults [1-3].

Fault detection methods have attracted significant attentions. It has become the main motivation in ensuring their system and product safe from further degradation. This required a good estimation of the current state. [4,5]

Unavoidable exposure to noisy environment, it is crucial to obtain a good estimation as possible, any misread information can lead to wrong control decision and worse, false alarm.

The use of Kalman filter in fault detection can be trace back since the introduction of the scheme itself. Since then many new variant has been developed to produce better approximation, such as Extended Kalman Filter (EKF) which its main purpose to overcome the KF in handling nonlinear system [6].

Even though EKF has been applied in many application, its drawback is well known [7,8]. A derivative free and point based filter have been proposed. One such example is Unscented Kalman Filter (UKF), which use the unscented transform to produce sigma point in its application [9].
Cubature Kalman Filter (CKF) is a later version of Kalman filter after UKF, it uses nearly as same principle as UKF but with better accuracy and computational efficiency $[10,11]$. It uses specifically generated cubature point using a combination of 3rd degree spherical and 3rd degree radial integration, which is later than propagated into the system model to produce a better estimation [12].

After its introduction, further improvement on CKF was done, such as algorithm corrections [13], adaptability [14] and root mean square [15]. However, the extent of its enhancement can only reach 3rd degree accuracy. Theoretically, the higher the degree, the higher the accuracy.

This has been achieved, by using 5 th degree spherical rule and 5th degree radial, a 5th degree CKF was developed. The generation of the algorithm is based on multiple integration over hyper-spherical surface [16]. Motivated by this consideration, this research proposed on designing a fault detection scheme for nonlinear system using higher degree cubature Kalman filter (HDCKF).

The paper is organizing as follows. Section 2 provides the formulation for HDCKF is some brief explanations on conventional CKF. Section 3 will explain the numerical simulation of the fault detection for CSTR with the obtained results, and conclusions are presented in section 4. 


\section{PROBLEM FORMULATION}

Consider a nonlinear discrete time system with sensors faults as:

$$
\begin{gathered}
x_{k}=f\left(x_{k-1}, u_{k-1}\right)+v_{k-1} \\
y_{k}=h\left(x_{k}, u_{k}\right)+\tilde{y}+z_{k}
\end{gathered}
$$

where $x_{k}$ is the state of the dynamic system at discrete time $k, f($.$) and h($.$) are some known functions, u_{k}$ is the known control input, $y_{k}$ is the measurement, $\tilde{y}$ denotes the unknown fault vector for the sensors, $v_{k-1}$ and $z_{k}$ are independent process and measurement Gaussian noise sequences with zero means and covariance's $Q_{k-1}$ and $R_{k}$, respectively.

Since our scope for this work is to measure the performance of HDCKF ability to detect fault, we only consider the problem of sensor fault detection.

Preparatory to the presentation of the fault detection mechanism, we review the conventional CKF to estimate the nonlinear model system state

\subsection{Cubature Kalman Filter (CKF)}

The step listed below is the Bayesian filtering framework which is required in implementing the 3rd degree $\mathrm{CKF}$, the different between a HDCKF lies in the cubature points generation in equation (4) and (9) [17,18].

\section{Time Update}

1)Assume at time $\mathrm{k}$, that the posterior density function $p\left(x_{k-1} \mid D_{k-1}\right)=N\left(\hat{x}_{k-1 \mid k-1}, P_{k-1 \mid k-1}\right)$ is known.

Factorize

$$
P_{k-1 \mid k-1}=S_{k-1 \mid k-1} S_{k-1 \mid k-1}^{T}
$$

2) Evaluate the cubature points $(\mathrm{i}=1,2, \ldots, \mathrm{m})$,

$$
X_{i, k-1 \mid k-1}=S_{k-1 \mid k-1} \xi_{i}+\hat{x}_{k-1 \mid k-1}
$$

where $m$ is number of cubature points based on CKF degree and $\xi_{i}$ is cubature point generator.

3) Evaluate the propagated cubature points $(i=1,2, \ldots, m)$,

$$
X_{i, k \mid k-1}^{*}=f\left(X_{i, k-1 \mid k-1}, u_{k-1}\right)
$$

4) Estimate the predicted state

$$
\hat{x}_{k \mid k-1}=\sum_{i=1}^{m} w_{i} X_{i, k \mid k-1}^{*}
$$

where $w_{i}$ is the specific weight for specific cubature points

5) Estimate the predicted error covariance

$$
P_{k \mid k-1}=\sum_{i=1}^{m} w_{i}\left(X_{i, k \mid k-1}^{*} X_{i, k \mid k-1}^{* T}-\hat{x}_{k \mid k-1} \hat{x}_{k \mid k-1}^{T}\right)+Q_{k-1}
$$

Measurement Update

1) Factorize

$$
P_{k \mid k-1}=S_{k \mid k-1} S_{k \mid k-1}^{T}
$$

2) Evaluate the cubature points $(i=1,2, \ldots, m)$,

$$
X_{i, k \mid k-1}=S_{k \mid k-1} \xi_{i}+\hat{x}_{k \mid k-1}
$$

3) Evaluate the propagated cubature points $(i=1,2, \ldots, m)$,

$$
Y_{i, k \mid k-1}=h\left(X_{i, k \mid k-1}, u_{k}\right)
$$

4) Estimate the predicted measurement

$$
\hat{y}_{k \mid k-1}=\sum_{i=1}^{m} w_{i} Y_{i, k \mid k-1}
$$

5) Estimate the innovation covariance matrix

$$
P_{z z, k \mid k-1}=\sum_{i=1}^{m} w_{i}\left(Y_{i, k \mid k-1} Y_{i, k \mid k-1}^{T}-\hat{y}_{k \mid k-1} \hat{y}_{k \mid k-1}^{T}\right)+R_{k}
$$

6) Estimate the cross-covariance matrix

$$
P_{x z, k \mid k-1}=\sum_{i=1}^{m} w_{i}\left(X_{i, k \mid k-1} Y_{i, k \mid k-1}^{T}-\hat{x}_{k \mid k-1} \hat{y}_{k \mid k-1}^{T}\right)
$$

7) Estimate the Kalman gain

$$
W_{k}=P_{x z, k \mid k-1} P_{z z, k \mid k-1}^{-1}
$$

8) Estimate the updated state

$$
\hat{x}_{k \mid k}=\hat{x}_{k \mid k-1}+W_{k}\left(y_{k}-\hat{y}_{k \mid k-1}\right)
$$

9) Estimate the corresponding error covariance

$$
P_{k \mid k}=P_{k \mid k-1}-W_{k} P_{z z, k \mid k-1} W_{k}^{T}
$$

\subsection{Higher Degree Cubature Kalman Filter (HDCKF)}

The Higher Degree Cubature Kalman Filter (HDCKF) exploit the use of multiple integral using Spherical and Radial rule. To compute the integral, transformation is required from Cartesian coordinate system to sphericalradial system. Details of the transformation have been presented in many articles $[19,20]$, and it is omitted from this paper. For any arbitrary function $f(x)$, with $x \in \mathbb{R}^{n}$ integral

$$
I(f)=\int_{R^{n}} f(x) \exp \left(-x^{T} x\right) d x
$$

can be decomposed to two integrals as

$$
I(f)=\frac{1}{\sqrt{\pi^{n}}} \int_{r=0}^{\infty} \int_{U_{n}} f(r s) r^{n-1} \exp \left(-r^{2}\right) d \sigma(s) d r
$$

Where $r$ represents the radius and $U_{n}$ is the surface of a unit sphere with dimension $n$. And further approximated by

$$
I(f) \approx \frac{1}{\sqrt{\pi^{n}}} \sum_{i=1}^{N r} \sum_{j=1}^{N s} w_{i} w_{j} f\left(\sqrt{2} r_{i} s_{j}\right)
$$

Where $N_{r}$ is the number of radial points, $N_{s}$ is the number of spherical points, $w_{i}$ is the weightage for radial rule, $r_{i}$ is the radial rules points, $w_{j}$ is the weightage for spherical integral, $s_{j}$ is the specific spherical points.

To represent HDCKF, 5th degree CKF is used, it is combination of 5 th degree spherical rule and 5th degree radial rule. 5th degree spherical weight and points is given by.

$$
\begin{aligned}
& w_{e}=\frac{4-n}{2 n(2 n(n+2))} A_{n}, \quad w_{s}=\frac{4}{4 n(n+2)} A_{n} \\
& \left\{e=\left[\begin{array}{ll}
1 & \\
0 & \\
0 & \ldots \\
0 &
\end{array}\right], s=\left[\begin{array}{cc}
\frac{1}{\sqrt{2}} & \\
\frac{1}{\sqrt{2}} & \ldots \\
0 & \\
0 &
\end{array}\right], n=4\right.
\end{aligned}
$$

And 5th degree radial weights and points 


$$
\begin{gathered}
w_{r 1}=\frac{1}{(n+2)} \Gamma\left(\frac{n}{2}\right), \quad w_{r 2}=\frac{n}{2(n+2)} \Gamma\left(\frac{n}{2}\right) \\
\left\{\begin{array}{l}
r_{1}=0 \\
r_{2}=\sqrt{\left(\frac{n}{2}\right)+1}
\end{array}\right.
\end{gathered}
$$

Thus, substituting the obtained results into (19)

$$
\begin{aligned}
& I(f)=\frac{2}{n+2} f(0) \\
& +\frac{4-n}{2(n+2)^{2}} \sum_{j=1}^{n}\left(f\left(\sqrt{n+2} \cdot e_{j}\right)+f\left(-\sqrt{n+2} \cdot e_{j}\right)\right) \\
& +\frac{1}{(n+2)^{2}} \sum_{j=1}^{n(n-1) / 2}\left(f\left(\sqrt{n+2} \cdot s_{j}^{+}\right)+f\left(-\sqrt{n+2} \cdot s_{j}^{+}\right)\right. \\
& \left.+f\left(\sqrt{n+2} \cdot s_{j}^{-}\right)+f\left(-\sqrt{n+2} \cdot s_{j}^{-}\right)\right)
\end{aligned}
$$

The point sets of $s_{j}^{+}$and $s_{j}^{-}$are given below

$$
\begin{aligned}
& \left\{s_{j}^{+}\right\} \stackrel{\Delta}{=}=\left\{\sqrt{\frac{1}{2}}\left(e_{k}+e_{l}\right): k<l ; k, l=1,2, \ldots, n\right\} \\
& \left\{s_{j}^{-}\right\} \stackrel{\Delta}{=}\left\{\sqrt{\frac{1}{2}}\left(e_{k}-e_{l}\right): k<l ; k, l=1,2, \ldots, n\right\}
\end{aligned}
$$

\subsection{Fault Detection Using HDCKF}

The fault detection considered in this research exploit the use of residual. Residual is the error value obtain between the estimated system state and the measurement. If there is no fault the result should be close to zero.

$$
g_{k}=y_{k}-\hat{y}_{k}=0
$$

where $\hat{y}_{k}$ is the estimated measurement. This signal should deviate from zero (zero mean) when a fault occurs i.e. $r_{k} \neq 0$

\section{NUMERICAL SIMULATION}

The CSTR system model is presented below $[21,22]$.

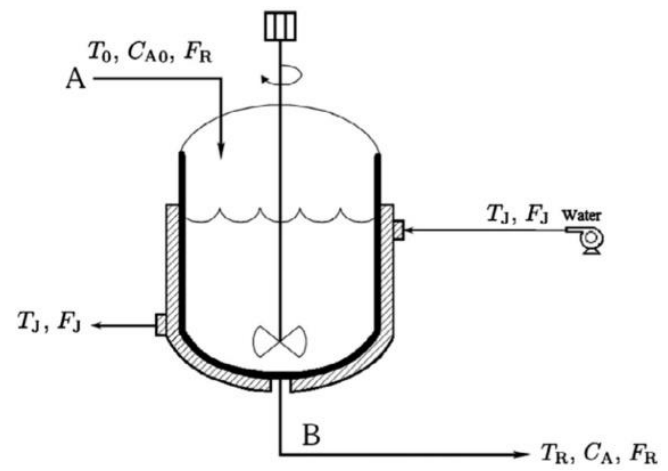

Figure 1. Non adiabatic continuous stirred tank reactor
Figure1 illustrates the CSTR, and the formula is reduced to a normalized dimensionless as:

$$
\begin{aligned}
& \frac{d x_{1}}{d t}=p_{1} u_{1}+p_{1} u_{1} u_{2}-p_{1} u_{2} x_{1} \\
& \quad-p_{2} e^{-p_{3} /\left(1+x_{2}\right)}\left(1+x_{1}\right)-p_{1} x_{1} \\
& \frac{d x_{2}}{d t}=p_{1} u_{3}+p_{1} u_{2} u_{3}-p_{1} x_{2}-p_{1} u_{2} x_{2} \\
& -p_{4} x_{2}+p_{4} x_{3}+p_{5} p_{2} e^{-p_{3} /\left(1+x_{2}\right)}\left(1+x_{1}\right) \\
& \frac{d x_{3}}{d t}=p_{6} x_{2}-p_{6} x_{3}-p_{7} x_{3}+p_{7} x_{4} \\
& \frac{d x_{4}}{d t}=p_{8} u_{4}+p_{8} u_{4} u_{5}-p_{8} x_{4}-p_{8} u_{5} x_{4}+p_{9} x_{3}-p_{9} x_{4}
\end{aligned}
$$

The parameter for the given formula system is as follows. $p_{1}=3.333 \times 10^{-2}, p_{2}=4.08 \times 10^{7}, p_{3}=25.347, p_{4}=6.63 \times 10^{-1}$, $p_{5}=1.45, p_{6}=5.97, p_{7}=5.97, p_{8}=1.67 \times 10^{-1}, p_{9}=1.33$. Input is given by $u=[1,1,1,1,1]^{T}$. The measurement matrix is represented as $y=C x$ with

$$
C=\left[\begin{array}{llll}
1 & 0 & 0 & 0 \\
0 & 1 & 0 & 0 \\
0 & 0 & 0 & 1
\end{array}\right]
$$

The noise value is increased to $v_{k}=0.01$ and $z_{k}=0.01$ to simulate harsh environment. The fault considered here is a sensor fault, appeared at $t>100$, taking place at concentration A sensor $(x l)$.
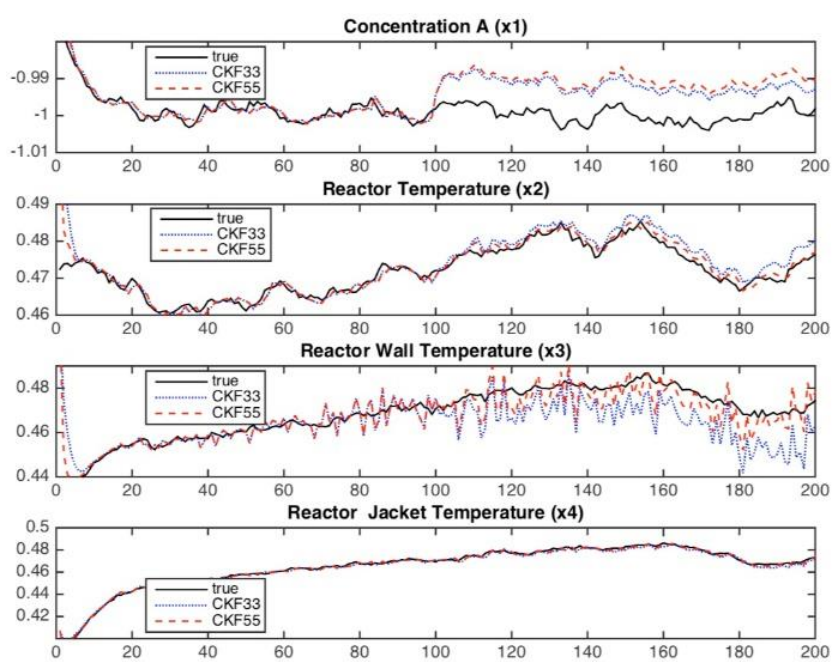

Figure 2. Fault detection using CKF and HDCKF

The fault detection using CKF and HDCKF estimator is illustrated in Figure2. It can be seen that HDCKF have a better estimation of reactor wall temperature.

From Figure 3 it is clear that the proposed HDCKF and CKF are able to detect occurred fault. Most importantly HDCKF show a much higher signal as compared to conventional $\mathrm{CKF}$, which indicates a much significant performance in identifying the fault. This resulted from HDCKF having more points than CKF, but it increased the computational time for the proposed filter. Table 1 shows 
the performance evaluation of CKF and HDCKF in term of RMSE, time and generated points.

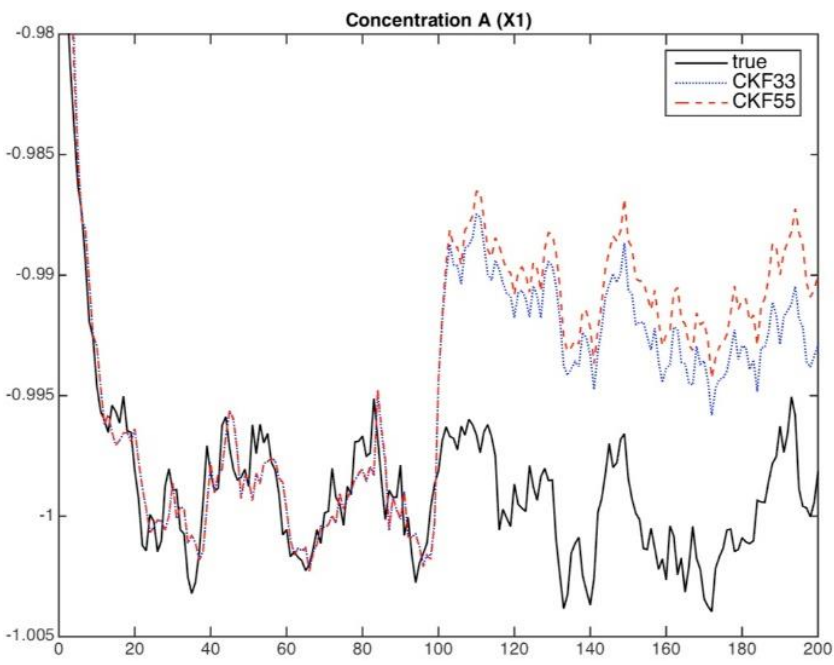

Figure 3. Fault detection using CKF and HDCKF for concentration A, fault occur at time $t>100$.

Table 1. Filters Performance Evaluation

\begin{tabular}{lccc}
\hline \multirow{2}{*}{ Filters } & \multicolumn{3}{c}{ Performance Evaluation } \\
\cline { 2 - 4 } & RMSE & Time (s) & Points \\
\hline CKF & 0.0015902 & 1.1331 & 8 \\
HDCKF & 0.0007476 & 2.8583 & 25 \\
\hline
\end{tabular}

\section{CONCLUSION}

In this paper, a fault detection using Higher Degree Cubature Kalman Filter is developed for a CSTR. The aim of the design is to obtain a higher accuracy of the state with and without the presents of fault. It is necessary to achieve this as it produce good fault detection residual. Simulation proves HDCKF performs better than CKF fault detection.

\section{REFERENCES}

[1] F.Pierri, G.Paviglianiti, F.Caccavale, M.Mattei. "Observer-based sensor fault detection and isolation for chemical batch reactors". Engineering Applications of Artificial Intelligence, vol. 21, no. 8, pp 1204-1216, 2008.

[2] B.Khajali, J.Poshtan. “ Fault Detection of a CSTR Using and Observer-Based Method and Particle Filters.” Journal of Control and System Engineering, vol 1, pp 30-36, 2013.

[3] Y.Chetouani. "Design of A Multi-Model ObserverBased Estimator for Fault Detection and Isolation (FDI) Strategy: Application to A Chemical Reactor." Brazilian Journal of Chemical Engineering, vol. 25, no. 4, pp 777-788, 2008.

[4] M. Du, J. Scott, P. Mhaskar. "Actuator and sensor fault isolation of nonlinear process systems." Chemical Engineering Science 104. 2013. 294-303

[5] Y.Zhang, X.R.Li. "Detection and Diagnosis of Sensor and Actuator Failures Using IMM
Estimator." IEEE Transactions on Aerospace and Electronic System, vol. 34, no. 4, 1998.

[6] S.Bahmanpour, M.Bashooki, and M.H.Refan. "State estimation and fault diagnosis of industrial process by using of particle filters." Proceeding 6th WSEAS International Conference on Signal Processing, Greece, pp 208-213, 2007.

[7] I. Arasaratnam, S. Haykin, and R.J. Elliott. "Discrete-time nonlinear filtering algorithms using Gauss-Hermite quadrature.” Proc. IEEE., vol. 95, no. 5, pp. 953-977, 2007.

[8] S. J. Julier and J. K. Uhlmann. "Unscented filtering and nonlinear estimation." Proc. IEEE, vol. 92, no. 3, pp. 401-422, Mar. 2004.

[9] K.Xiong, C.Chan, H.Zhang. "Detection of Satellite Attitude Sensor Faults Using The UKF." IEEE Transactions on Aerospace and Electronic Systems, Vol. 43, no. 2, pp. 480-491, 2007.

[10] I. Arasaratnam and S. Haykin. 2009. "Cubature Kalman filters.” IEEE Trans. Autom. Control, vol. 54, no. 6, 2009. 1254-1269.

[11] S.Y. Wang, J.C. Feng, and C.K. Tse. "Spherical simplex-radial cubature Kalman filter." IEEE Signal Process. Lett., vol. 21, no. 1, pp. 43-46, 2014

[12] B. Shovan and Swati. "Square-root CubatureQuadrature Kalman Filter." Asian Journal of Control, vol. 16, no. 2, pp. 617-622, 2014.

[13] X. Tang, J. Wei, K. Chen. "Square-Root adaptive Cubature Kalman Filter with application to spacecraft attitude estimation." 15th International Conference on Information Fusion (FUSION), (012.1406-1412.

[14] J. Zarei, E. Shokri, and H.R. Karimi. "Convergence analysis of Cubature Kalman filter." in European on Control Conference (ECC), 2014, pp. 1367-1372.

[15] W.Li, Y.Jia. 2012. "Location of mobile station with maneuvers using an IMM-based Cubature Kalman Filter." IEEE Transactions on Industry Electronics 59 11. 2012. 4338-4348

[16] B. Shovan and Swati. "Cubature Quadrature Kalman Filter.” IET Signal Processing, vol. 7, no. 7, pp. 533541,2013

[17] B. Jia, M. Xin, and Y. Cheng. 2013. "High-degree Cubature Kalman Filter." Automatica, vol. 49, no. 2, 2013. 510-518.

[18] S.Zhang, M.Baric. "A Bayesian Approach to Hybrid Fault Detection and Isolation." IEEE 54th Annual Conference on Decision and Control (CDC), 2015

[19] W.Wang, X.Chen. "Application of Improved 5th Cubature Kalman Filter in Initial Strapdown Inertial Navigation System Alignment for Large Misalignment Angles.” Sensors 2018. 18. 659

[20] Z.Li, W.Yang, D.Ding, Y.Liao, “A Novel Fifth Degree Cubature Kalman Filter for Real Time Orbit Determination by Radar." Mathematical Problems In Engineering 2017.9

[21] J.Zarei, E.Shokri. "Robust sensor fault detection based on nonlinear unknown input observer." Measurement, vol. 48, pp 355-367,.2014.

[22] J. Zarei, J. Poshtan. "Design of nonlinear unknown input observer for process fault detection." Industrial \& Engineering Chemistry Research 49 22. 2010. 11443-11452. 